\title{
High-Field Low-Frequency Spin Dynamics
}

\author{
A.M. Farutin, V.I. Marchenko \\ P.L. Kapitza Institute for Physical Problems, RAS \\ 119334, Kosugina 2, Moscow, Russid*
}

(Dated: November 5, 2018)

\begin{abstract}
The theory of exchange symmetry of spin ordered states is extended to the case of high magnetic field. Low frequency spin dynamics equation for quasi-goldstone mode is derived for two cases of collinear and noncollinear antiferromagnets.
\end{abstract}

PACS numbers: 75.10.-b, 75.30.Ds, 75.30.Gw, 76.50.+g

Keywords: exchange symmetry, magnetic symmetry, spin dynamics, high-field spin ordering.

Low frequency spin dynamics exists in magnetic materials if spin-orbital and magnetic dipole-dipole effects are small compared to the exchange ones. The number of the quasi-goldstone modes and the spin dynamics equations are determined by the exchange symmetry of a given spin structure [1]. The concept of the exchange symmetry can be easily extended to the spin structures strongly deformed by high external magnetic field. In this case there is only one quasi-goldstone mode. It is related to the invariance of exchange and Zeeman energies upon the rotation of the spin space around magnetic field direction on some angle $\psi$. In this Letter we derive the low frequency spin dynamics equations for the two characteristic examples of the antiferromagnets.

From the symmetry point of view there is no difference between the description of the spin structures with field induced spin polarization, and those with spontaneous spin polarization (ferrimagnets). The classification of exchange spin structures is based on the concept of the exchange space symmetry group $G$. This is a group of the charge density symmetry in the exchange approximation (i.e., without relativistic effects).

The extension of the exchange symmetry concept in our case is straightforward. Nonetheless one should note that the only effect of the magnetic field which can be taken into account in the spin Hamiltonian is the Zeeman term $-\gamma \hat{\mathbf{S}} \mathbf{H}$. Here $\gamma$ is the free electron gyromagnetic ratio, and $\hat{\mathbf{S}}$ is the total spin operator. The magnetic field effect on the orbital state of electrons should be neglected. It is easy to check that this approximation is valid for all known magnets. Namely, the change of the orbital state remains small even at the spin-flip field.

The dynamics equation for quasi-goldstone degree of freedom $\psi$ can be derived from Lagrange formalism. The kinetic energy is

$$
K=\frac{I}{2} \dot{\psi}^{2}
$$

At low fields the moment of inertia $I=\gamma^{-2} \chi$, where $\chi$ is magnetic susceptibility (see [1]). One can generalize this result to the high fields. On one hand the magnetization

*farutin@kapitza.ras.ru, mar@kapitza.ras.ru
$\mathbf{M}$ is the derivative of Lagrange function with respect to magnetic field $\mathbf{M}=\partial L / \partial \mathbf{H}$. On the other hand in the exchange approximation the magnetization is $\mathbf{M}=\gamma \mathbf{S}$. The mechanical spin momentum is oriented along the magnetic field and its value is $S=\delta L / \delta \dot{\psi}$. Let's expand the Lagrange function on small $\dot{\psi}$

$$
L=A+B \dot{\psi}+\frac{I}{2} \dot{\psi}^{2}+\ldots
$$

The two definitions of magnetization give us the following relations

$$
M=\frac{\partial A}{\partial H}+\frac{\partial B}{\partial H} \dot{\psi}+\ldots=\gamma(B+I \dot{\psi}+\ldots) .
$$

The magnetization consists of the static $M_{s}=\partial A / \partial H=$ $\gamma B$, and dynamic $M_{d} \approx \partial B / \partial H \dot{\psi}=\gamma I \dot{\psi}$ parts. From these we see that the moment of inertia $I=\gamma^{-2} \tilde{\chi}$, where $\tilde{\chi}$ is the static differential susceptibility.

For the first case consider a collinear antiferromagnets. At high magnetic field the antiferromagnetic vector $\mathbf{L}$ is usually perpendicular to the field. As it is clear from general results of the exchange symmetry theory [1] the exchange crystal symmetry group $G$ remains the same as in the absence of magnetic field. The antiferromagnetic vector transforms according to one of the one-dimensional representations of the group $G$. Orientation of the vector $\mathbf{L}$ around the field is dictated by the crystal anisotropy.

Let us introduce Euler angles $\theta, \varphi, \psi$ between unit orthogonal basis $\mathbf{a}, \mathbf{b}, \mathbf{c}$ in the spin space and the unit orthogonal basis $\mathbf{x}, \mathbf{y}, \mathbf{z}$ in the orbital space. Let us chose the orientations of the basic vectors $\mathbf{c} \| \mathbf{H}$, and $\mathbf{a} \| \mathbf{L}$.

The part of the energy which depends on orientation of the antiferromagnetic vector $\mathbf{L}$ is

$$
U=\frac{\beta}{2} a_{z}^{2}+\frac{g_{i k}}{2} \partial_{i} \psi \partial_{k} \psi
$$

Here we consider the symmetry groups $G$ with one principal axis $C_{3}, C_{4}$, or $C_{6},\left(\mathbf{z} \| C_{n}\right)$. The tensor $g_{i k}$ is of an exchange nature $\left(g_{z z}=g_{\|}, g_{x x}=g_{y y}=g_{\perp}\right)$. The value $\beta$ corresponds to the relativistic effects of anisotropy. All the parameters of our theory such as $g_{i k}$, and $\beta$ remain unknown functions of the magnetic field value. However, the field orientation dependence of the spin dynamics will be determined. 
The energy (4) can be written in the form

$$
U=\frac{\beta}{2} \sin ^{2} \theta \cos ^{2} \psi+\frac{g_{i k}}{2} \partial_{i} \psi \partial_{k} \psi
$$

The Lagrange equation corresponding to the kinetic energy (11) and the potential energy (4) is

$$
I \ddot{\psi}=\beta \sin ^{2} \theta \cos \psi \sin \psi+g_{i k} \partial_{i} \partial_{k} \psi \text {. }
$$

The spin wave spectrum $\omega(q)$ is

$$
\omega=\sqrt{\omega_{0}^{2}+\frac{g_{i k}}{I} q_{i} q_{k}},
$$

where the gap $\omega_{0}$ for both possible types of the anisotropy $\beta>0$ (easy plane), and $\beta<0$ (easy axis) is

$$
\omega_{0}=\gamma \sqrt{\frac{|\beta|}{\tilde{\chi}} \mid} \sin \theta \mid .
$$

The gap is the longitudinal AFMR frequency. This statement comes from the expansion (2). The second term $B(H) \dot{\psi}$ is a full derivative for the constant field. But if the field is time dependent then this term leads to the description of the longitudinal AFMR.

If the field orientation is close to the main crystal axis $(\theta=0)$, the gap in the spectrum goes to zero. The finite gap value appears if one takes into account next relativistic terms of basic plane anisotropy.

Note, that one should add the Dzialoshinskii term $d\left(a_{x} c_{y}-a_{y} c_{x}\right)=d \sin \theta \sin \psi$ to the energy (4) for the antiferromagnets with a weak spontaneous ferromagnetism.

For the second case consider the noncollinear antiferromagnet $\mathrm{Mn}_{3} \mathrm{Al}_{2} \mathrm{Ge}_{3} \mathrm{O}_{12}$. Its AFMR spectrum was studied experimentally and theoretically at small fields in 2]. The twelve sublattices spin structure of $\mathrm{Mn}_{3} \mathrm{Al}_{2} \mathrm{Ge}_{3} \mathrm{O}_{12}$ is described in terms of two antiferromagnetic vectors $\mathbf{L}_{1} \perp \mathbf{L}_{2}$ which transform according to two-dimensional representation $E_{u}$ of the crystal class $O_{h}$.

As observed in [2] the spin plane tends to become perpendicular to the field. So, the most probable state of the spin structure at high fields is $\mathbf{L}_{1}, \mathbf{L}_{2} \perp \mathbf{H}$. Then, according to [1] the exchange space group remains $O_{h}^{10}$.

At high fields the anisotropy effects are described to a first relativistic approximation by the same invariant

$$
U_{a}=\beta\left\{\sqrt{3}\left(a_{z}^{2}-b_{z}^{2}\right)+2\left(a_{x} b_{x}-a_{y} b_{y}\right)\right\}
$$

as in the small field limit (see [2]). Let us align the basic vectors $\mathbf{x}, \mathbf{y}, \mathbf{z}$ in the orbital space along fourthorder symmetry axes. The basis in the spin space is chosen as $\mathbf{c}\|\mathbf{H}, \mathbf{a}\| \mathbf{L}_{1}$, and $\mathbf{b} \| \mathbf{L}_{2}$. In terms of Euler angles the anisotropy energy (9) can be rewritten as $\beta f \cos 2\left(\psi-\psi_{0}\right)$, where parameters $f$, and $\psi_{0}$ are the functions of the field orientation

$$
\begin{gathered}
f=\sqrt{\cos ^{2} 2 \varphi\left(1+\cos ^{2} \theta\right)^{2}+\left(\sqrt{3} \sin ^{2} \theta+2 \sin 2 \varphi \cos \theta\right)^{2}}, \\
\tan 2 \psi_{0}=\frac{\cos 2 \varphi\left(1+\cos ^{2} \theta\right)}{\sqrt{3} \sin ^{2} \theta+2 \sin 2 \varphi \cos \theta} .
\end{gathered}
$$

The magnon spectrum here is given by the general formula (7), where $g_{i k}=g \delta_{i k}$, and

$$
\omega_{0}=2 \gamma \sqrt{\frac{2|\beta| f}{\tilde{\chi}}} .
$$

The anisotropy term (9) is the main relativistic correction to the exchange energy of $\mathrm{Mn}_{3} \mathrm{Al}_{2} \mathrm{Ge}_{3} \mathrm{O}_{12}$. This fact leads to a new relation between static and dynamic characteristics. Consider anisotropic correction to the magnetization. The ground state anisotropy energy is $U_{a}=-4|\beta|$ if the magnetic field is oriented along [001] axis $(\theta=0)$, and $U_{a}=-16|\beta| / 3$ if it is oriented along [111] $(\cos \theta=1 / \sqrt{3}, \varphi=\pi / 4)$. It gives an opportunity to find the function $\partial \beta / \partial H$. Namely,

$$
\frac{\partial|\beta|}{\partial H}=\frac{3}{4}\left(M_{[111]}-M_{[001]}\right) .
$$

The obtained results can be applied to any usual magnetics, as well as to tensors spin structures 3]. In all cases there are additional relations of type (11). But, the situations can be more complicated. It may become necessary to take into account small deviations of the magnetization from the field orientation.

In conclusion, we have presented the exchange symmetry theory of spin ordered states strongly deformed by high magnetic field. Spin dynamics equation is derived for quasi-goldstone mode which is the rotation of spin space around the field direction. The dispersion of the spin waves is determined. A new relation between static and dynamic characteristics is found.

We thank A.F. Andreev, L.A. Prozorova, and A.I. Smirnov for helpful discussions. The work is supported by RFBR Grants No. 04-02-17294, 06-02-16509, and 0602-17281, RF President Program, and Landau Scholarship (A.F.) from Forschungszentrum Juelich.
[1] A.F. Andreev and V.I. Marchenko, Sov. Phys. Uspekhi. 23, 21 (1980)

[2] L.A. Prozorova, V.I. Marchenko, and Yu.V. Krasnyak,
JETP Lett. 41, 637 (1986)

[3] A.M. Farutin and V.I. Marchenko, JETP. 100, 977 (2005) 